\title{
Grand Unified String Theories with SU(3) Gauge Family Symmetry
}

\author{
A.A. Maslikov ${ }^{b}$, G.G. Volkov ${ }^{a, b}{ }^{1}$ \\ (a) INFN Sezione di Padova \\ and Dipartimento di Fisica Universitì di Padova, \\ Via Marzolo 8, 35100 Padua, Italy. \\ (b) Institute for High Energy Physics, \\ 142284 Protvino, Moscow Region, Russia
}

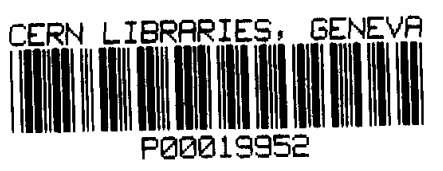

\begin{abstract}
In the framework of four dimensional heterotic superstring with free fermions we investigate the rank eight Grand Unified String Theories (GUST) which contain the $S U(3)_{H}$-gauge family symmetry. GUSTs of this type accomodate naturally three fermion families presently observed and, moreover, can describe fermion mass spectrum without high dimensional representations of conventional unification groups. We explicitly construct GUST with gauge symmetry $G=S U(5) \times U(1) \times(S U(3) \times U(1))_{H} \subset S O(16)$ in free complex fermion formulation. As the GUSTs originating from Kac - Moody algebras (KMA) contain only low-dimensional representations it is usually difficult to break the gauge symmetry. We solve this problem taking for the observable gauge symmetry the diagonal subgroup $G^{\text {sym }}$ of rank 16 group $G \times G \subset S O(16) \times S O(16) \subset E(8) \times E(8)$. Such a construction effectively corresponds to a level two $\mathrm{KMA}$, and therefore some higher dimensional representations of the diagonal subgroup appear. This (due to $G \times G$-tensor Higgs fields) allows one to break GUST symmetry down to the $S U\left(3^{c}\right) \times U(1)_{e m}$. In this approach the observed electromagnetic charge $Q^{e m}$ can be viewed as a sum of two $Q^{I}$ - and $Q^{I I}$ charges of each G- group. In this case below the scale where $G \times G$ breaks down to $G^{\text {symm }}$ the spectrum does not contain particles with exotic fractional charges.
\end{abstract}

\footnotetext{
'E-mail:volkov_g@mx.ihep.su
}

UNIVERSITA I)EGLI STUDI DI PADOVA

DIPARTIMENTO DI FISICA "GALILEO GALILEI"

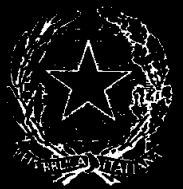

ISTITUTO NAZIONALE DI FISICA NUCLEARE

SEZIONE DI PADOVA 

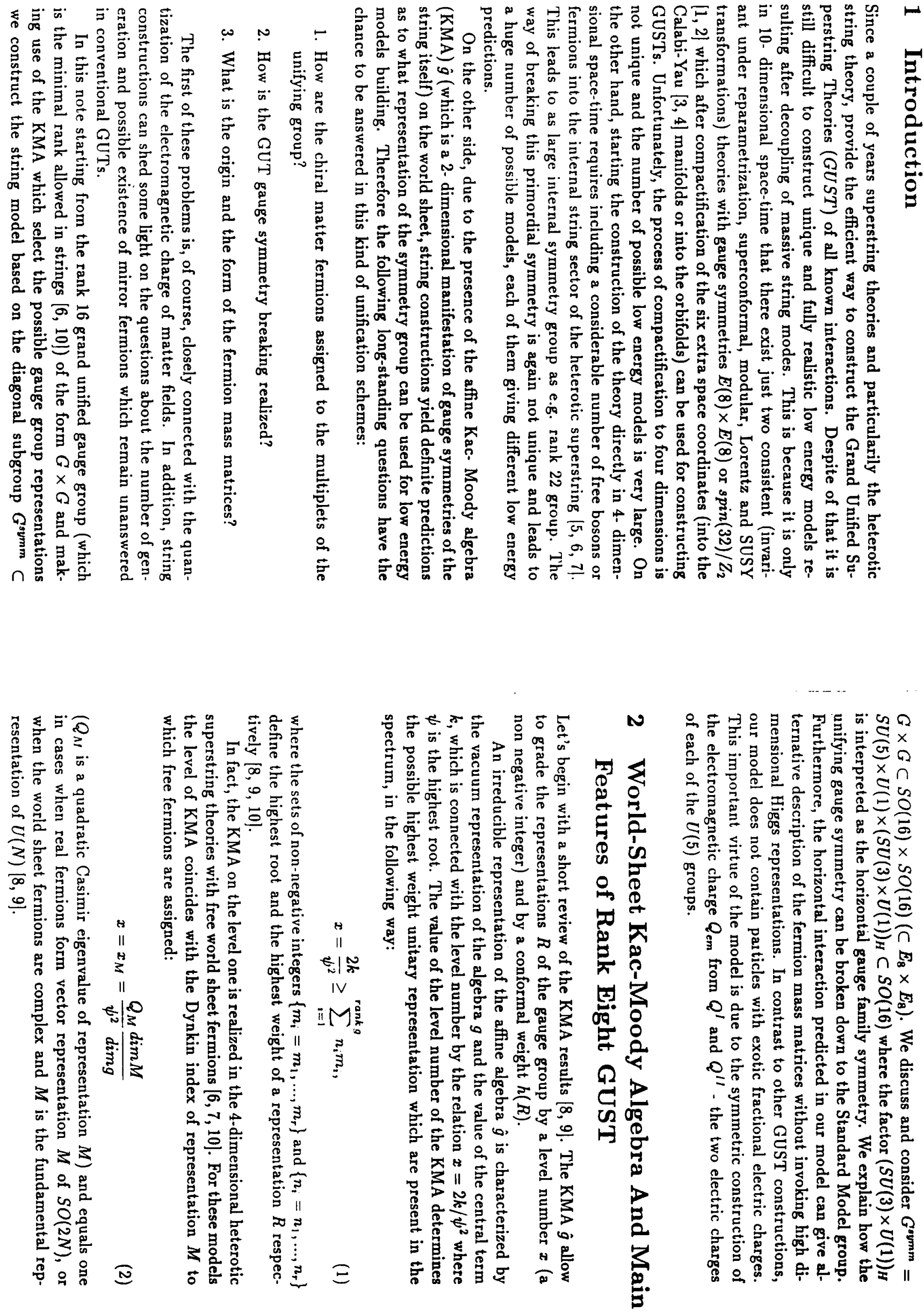


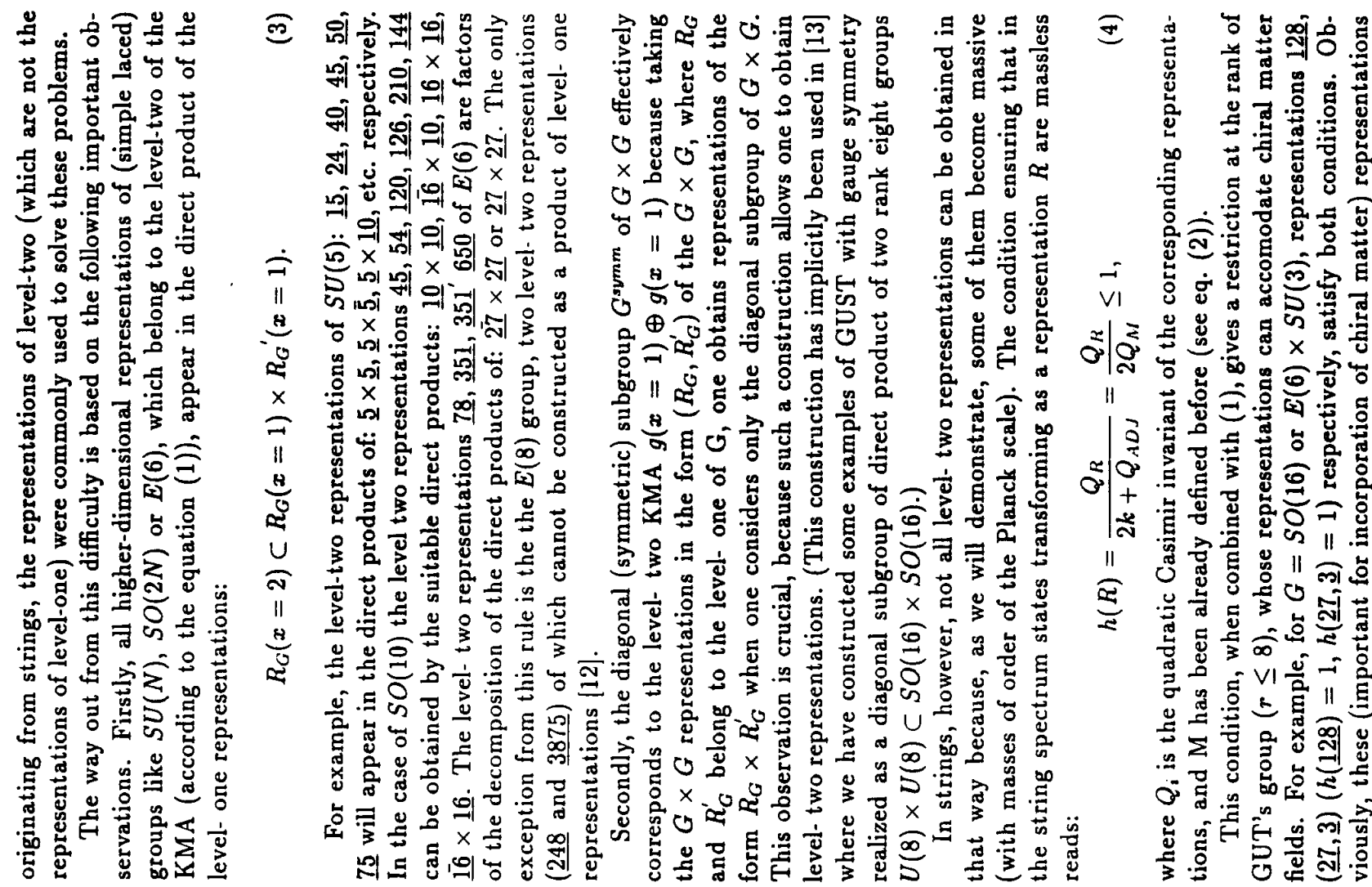

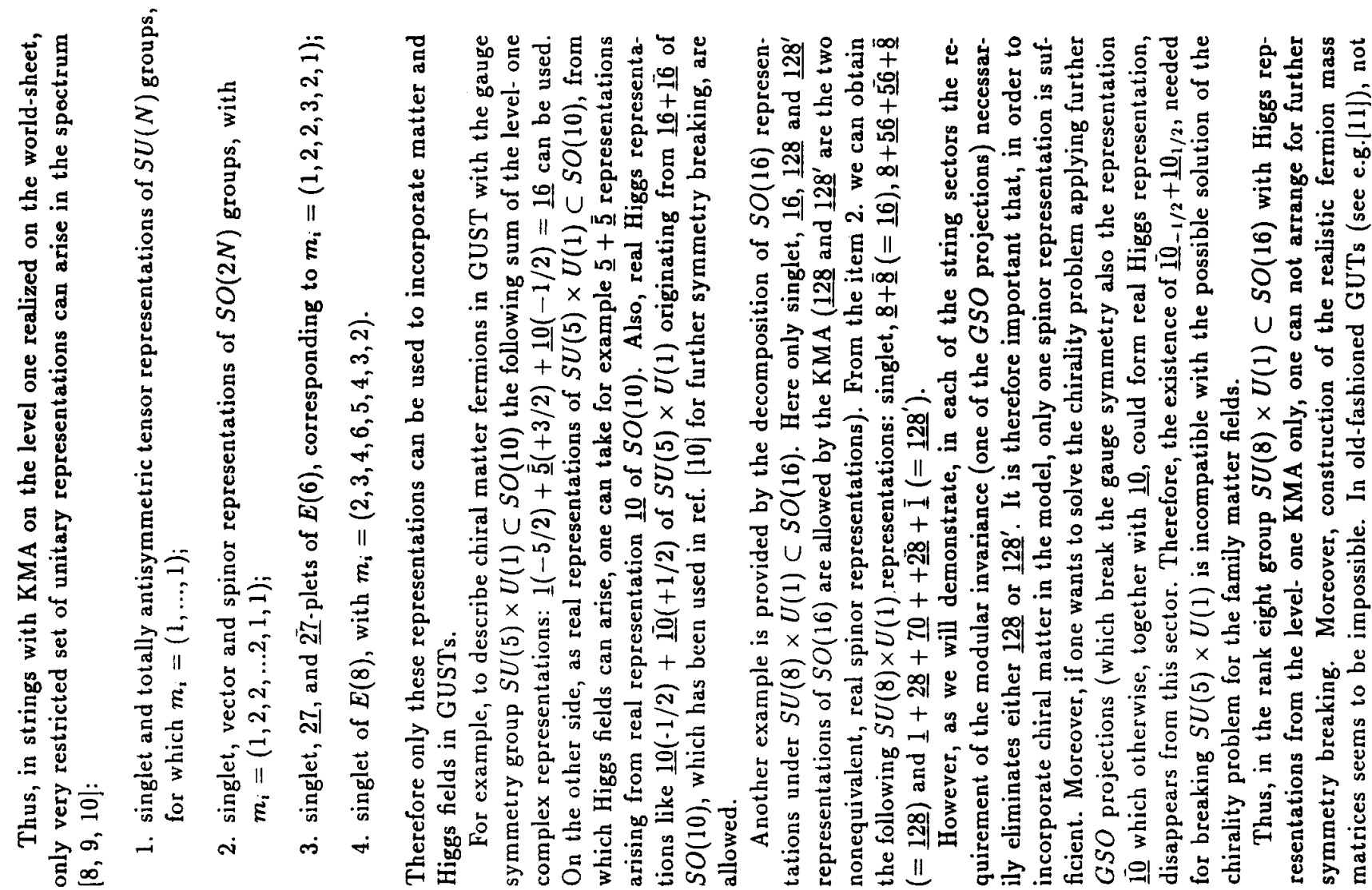




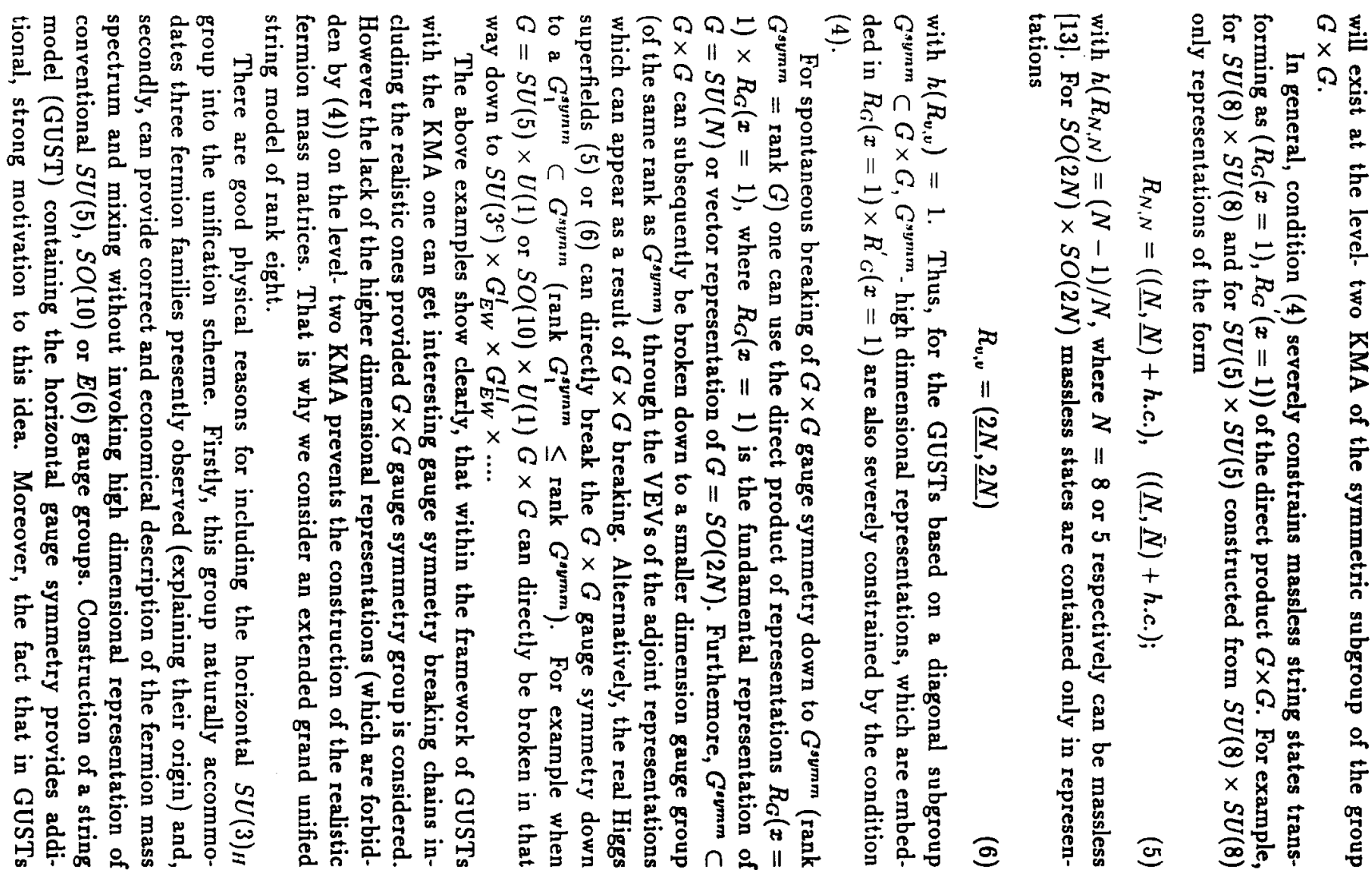

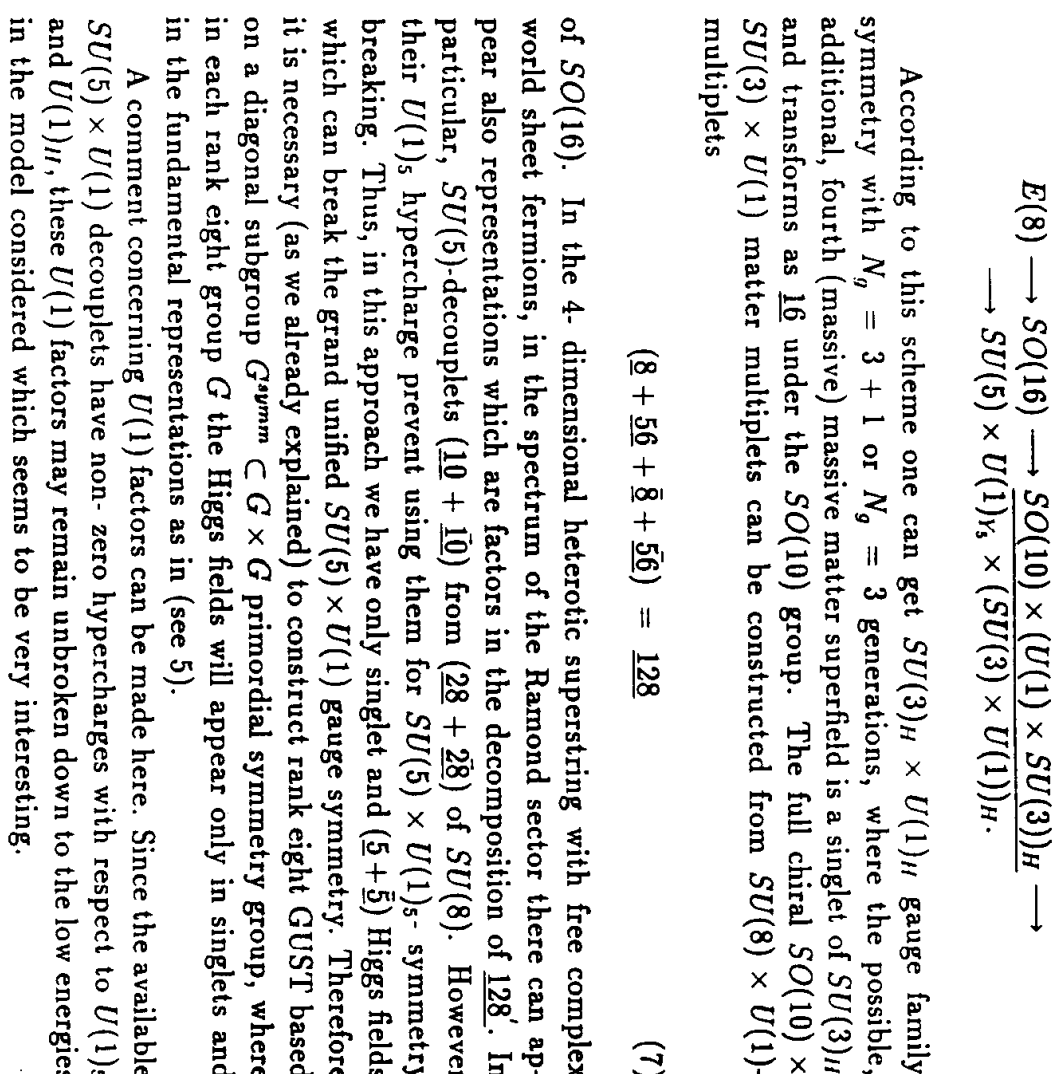

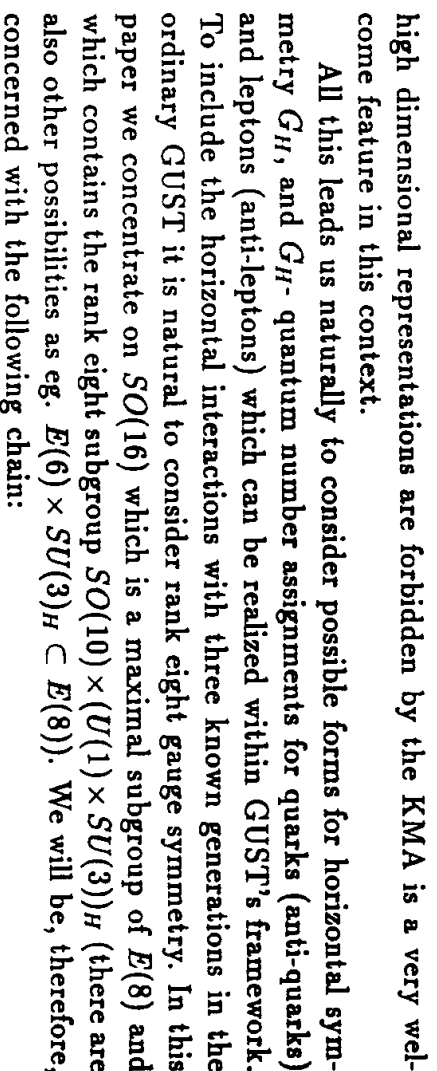



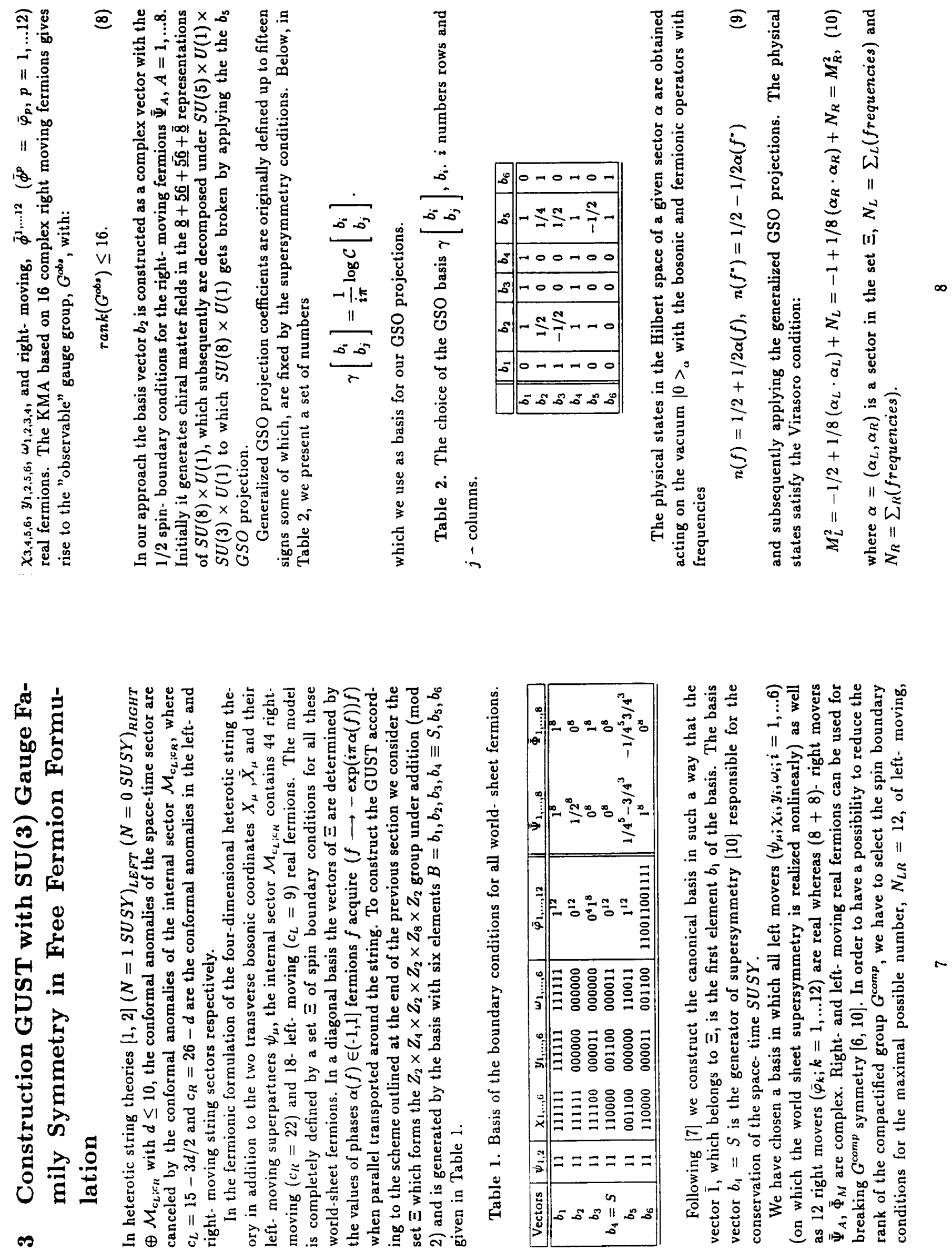

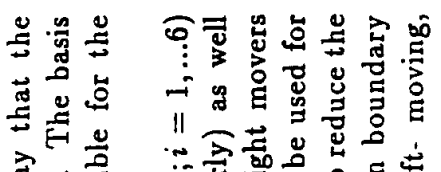

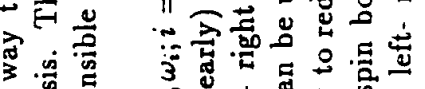

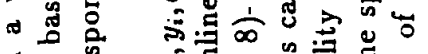

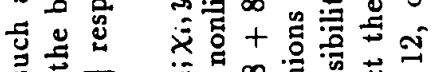

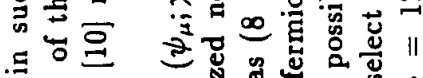

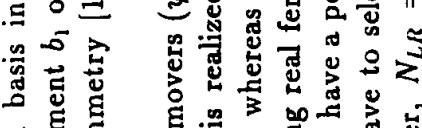

范焉

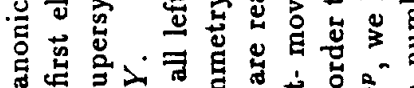

造动

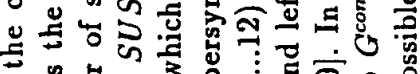

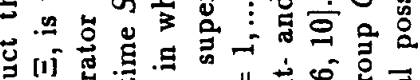

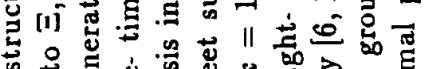

品

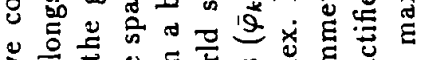

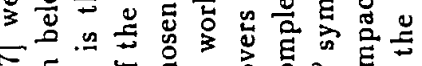

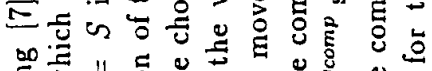

.

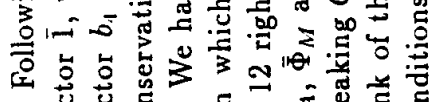

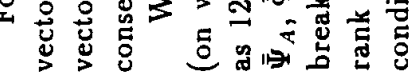



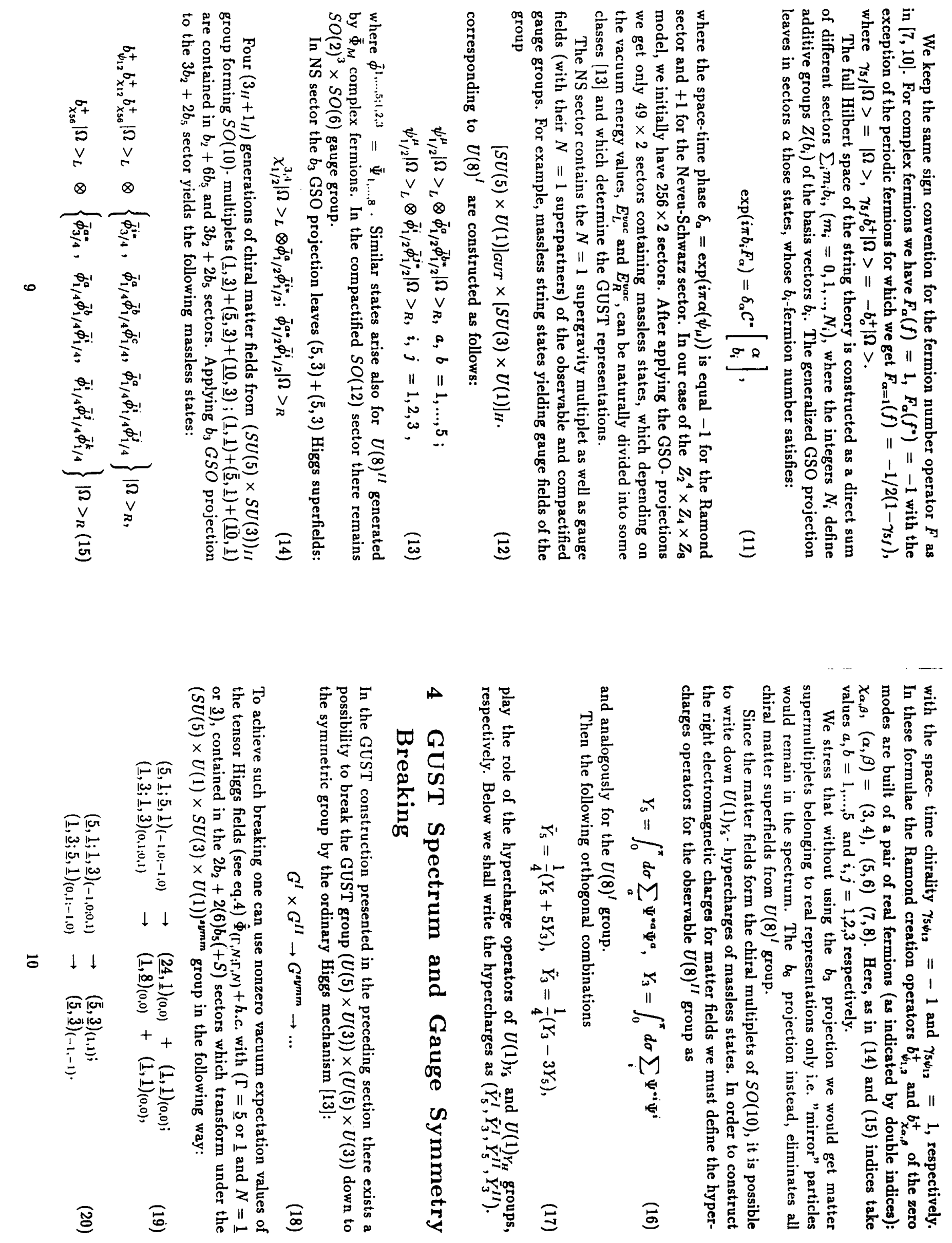

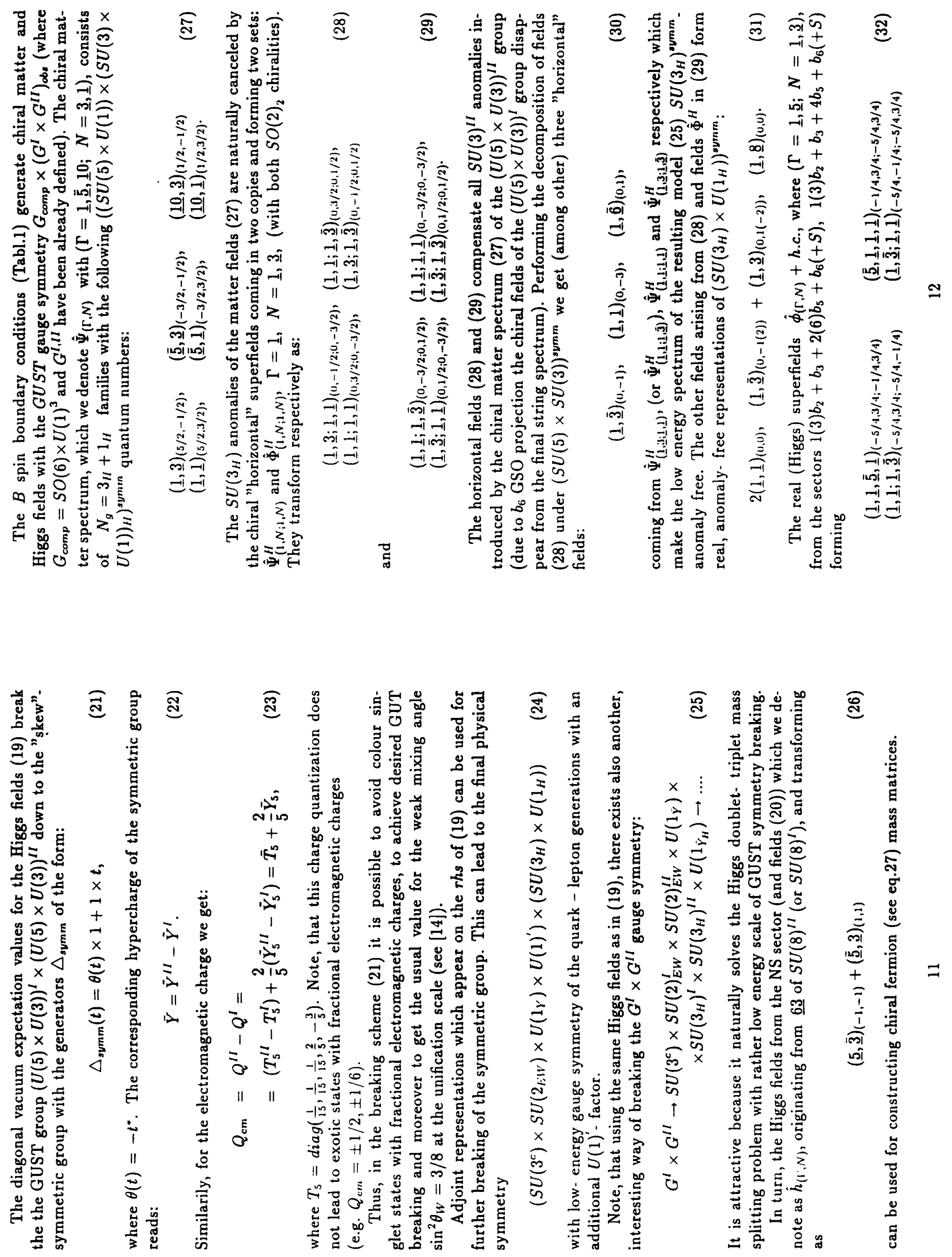

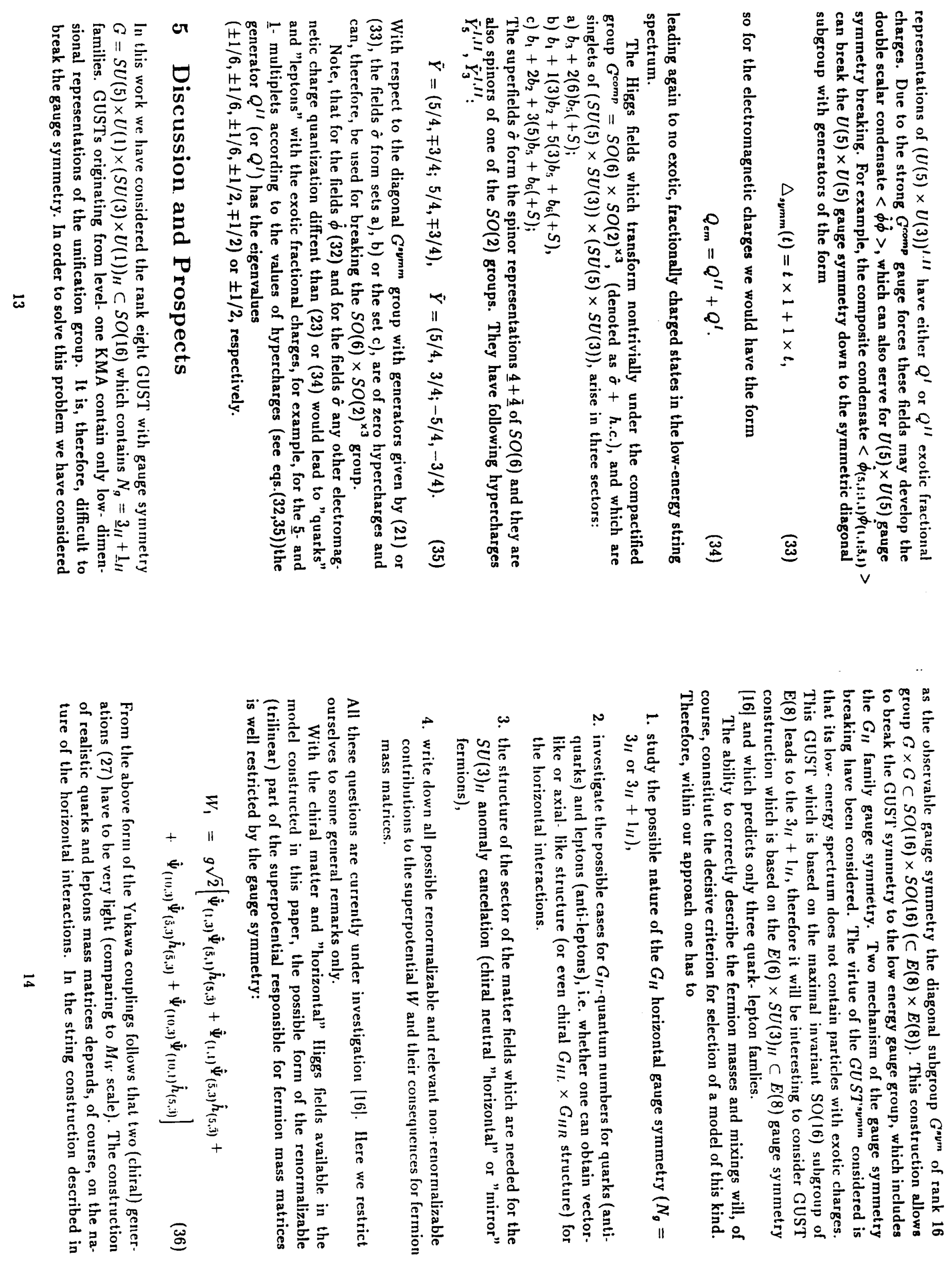

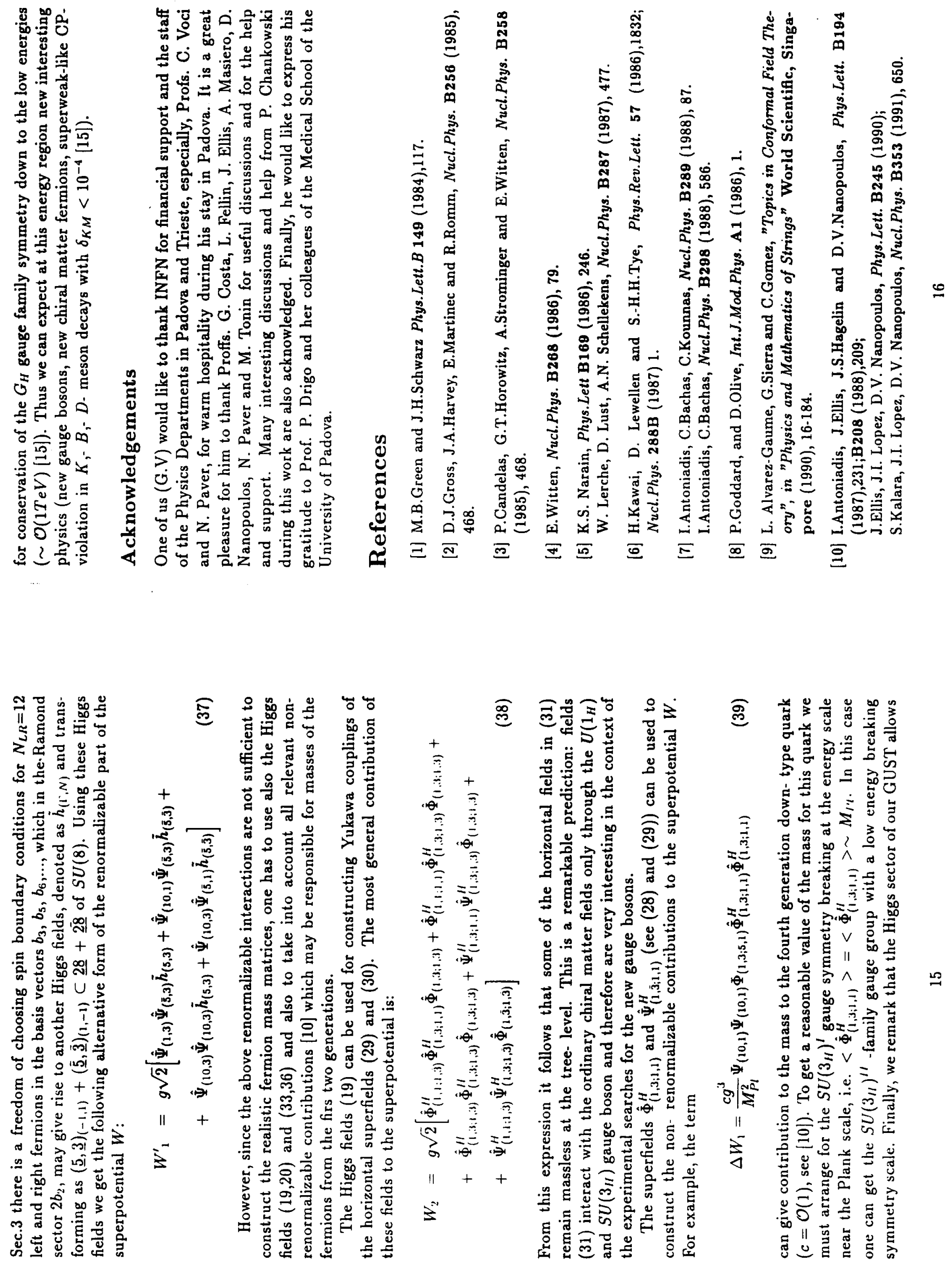


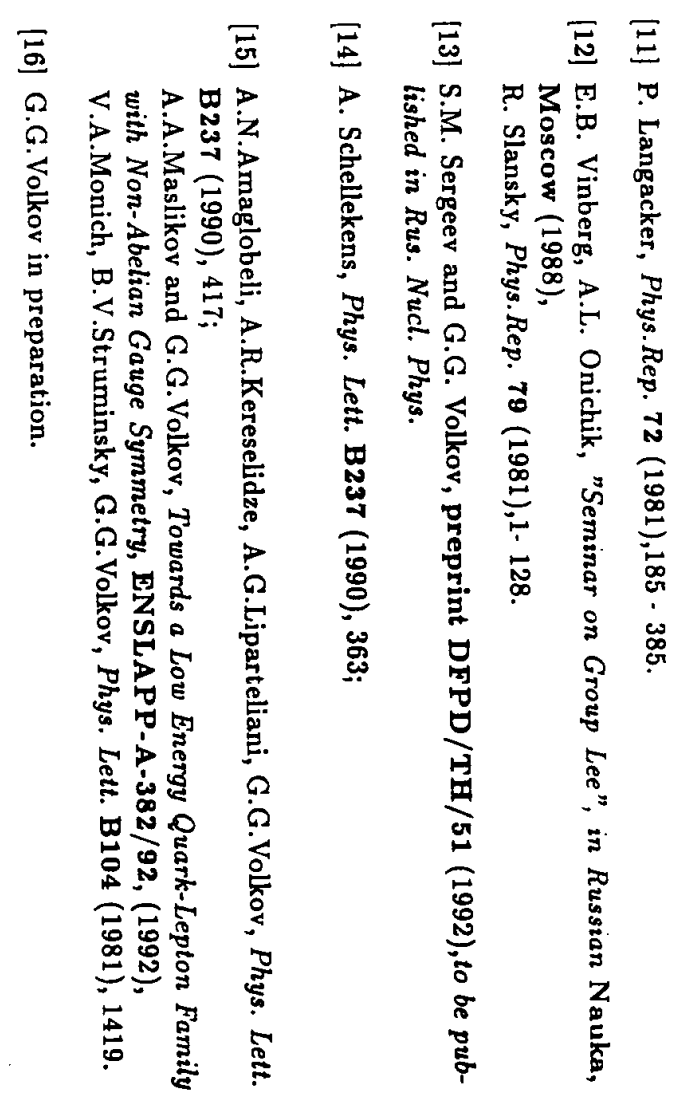


